\title{
Fusion-fission studies on ${ }^{19} \mathbf{F}+{ }^{206,208} \mathbf{P b}$ and ${ }^{11} \mathbf{B},{ }^{19} \mathbf{F}+{ }^{238} \mathbf{U}$ reactions around bar- rier energies at IUAC facility
}

\author{
Sugathan Pullanhiotan ${ }^{1}{ }^{\star}$, Rakesh Dubey ${ }^{1}$, Chandrabhan Yadav ${ }^{1}$, Akhil Jhingan $^{1}$, Golda Komalan Satheedas ${ }^{1}$, Sa- \\ neesh Nedumbally ${ }^{1}$, and Mohit Kumar ${ }^{1}$ \\ ${ }^{1}$ Inter University Accelerator Centre, Aruna Asaf Ali Marg, New Delhi, India 110067
}

\begin{abstract}
Fission process is strongly influenced by entrance channel dynamical variables. Among these, the nuclear charge product, mass asymmetry and deformation play important role in fission dynamics. Reaction characteristics are distinguished by investigating the properties of fission mass and angular distributions. Experiments using actinide targets are challenging due to many conflicting results making unambiguous identification of quasi-fission difficult. At IUAC accelerator facility many experiments have been performed to make a systematic study of fission mechanism and role of entrance channel parameters and deformation. Fragment mass distribution, angular distribution and neutron multiplicity measurements are performed to study reactions using spherical and deformed targets.
\end{abstract}

\section{Introduction}

One of the key problems in heavy ion collision is to understand what are the important variables governing the dynamics of colliding heavy nuclei at various energies. In collisions where heavy partners are involved, it is observed that quasi-fission $(\mathrm{QF})$ is a competing process that hinders complete fusion and subsequent formation of residues of heavy nuclei. At high excitation energies process like fast fission dominates and their contribution found to be negligible at moderate excitation energies. The dynamics of the fusion process is strongly influenced by many entrance channel properties such as charge product of nuclei $\left(Z_{p} Z_{t}\right)$, mass asymmetry $(\alpha)$, deformation of nuclei $(\beta)$, shell effects and collision energy[1-3]. Classically, QF has been observed in high fissile systems with $Z_{p} Z_{t}>1600$. However there are reaction systems reported where QF were observed in lighter system with $Z_{p} Z_{t} \sim 800$ [4]. Mass asymmetry and deformation found to be the factors influencing the onset of QF in such light systems. For example, the variance of the mass distribution of fission fragments found to be larger in ${ }^{19} \mathrm{~F}+{ }^{197} \mathrm{Au}$ and ${ }^{30} \mathrm{Si}+{ }^{186} \mathrm{~W}$ reactions as compared to ${ }^{12} \mathrm{C}+{ }^{204} \mathrm{~Pb}$, though all of them formed the same compound nucleus $(\mathrm{CN}){ }^{216} \mathrm{Ra}$ at similar excitation energies[4]. This was attributed to presence of quasi-fission in former two reactions whose entrance channel mass asymmetry $(\alpha)$ is lower than the critical mass asymmetry $\alpha_{B G}$ (Businaro Gallone mass asymmetry for maximum potential energy). It was reported that, more symmetric systems with mass asymmetry lower than the critical mass asymmetry evolves through QF process. On the other hand, asymmetric reactions with mass asymmetry larger than critical mass asymmetry leads to

\footnotetext{
^e-mail: sugathan@iuac.res.in
}

compact compound nucleus equilibrated in all degrees of freedom. Apart from mass asymmetry, deformation of the nuclei also played role in fission dynamics. Reactions involving deformed target showed anomalously large angular anisotropy and enhancement of mass variance at low energy[5-8]. The anomalously large mass variance and angular anisotropy below barrier energies are reported to be due to orientation dependent QF.

Experimental investigation of mass and angular distribution of fission products is the most commonly employed method for identifying compound and non-compound nuclear processes. According to standard statistical model of fission, the mass distribution of fission products follow Liquid Drop Model (LDM) predictions showing symmetric mass distribution and the width of mass distribution vary smoothly as a function of excitation energy. For angular distribution, the basic assumption is that $\mathrm{K}$ (the projection of angular momentum on symmetry axis) is fully equilibrated at the saddle point and a symmetric angular distribution indicates the decay from an equilibrated system. However for any non-compound process such as QF, mass asymmetry and $\mathrm{K}$ degree of freedom may not be fully equilibrated before the fission, resulting in large mass width and angular anisotropy deviating considerably from model predictions. Large angular anisotropies observed in fission involving actinide targets have been attributed to presence of either fission before $\mathrm{K}$ equilibration, i.e pre-equilibrium fission(PEF) or the quasi-fission[9-14]. Presence of QF signify less chance for evaporation residue(ER)formation and one expect reduced ER cross section in such reactions. Another distinct feature of QF is the strong mass angle correlation that has been clearly observed in systems involving heavy projectiles[1]. Though reason for large mass width and 
anisotropy have been attributed to QF, the identification of the reaction process is not always unambiguous. For example in reaction ${ }^{16} \mathrm{O}+{ }^{238} \mathrm{U}$ around barrier energies, large mass width and anisotropy have been observed, but no suppression of ER observed[3, 7, 15]. So unambiguous identification of QF events becomes a challenging task, especially when lighter projectiles are involved in low $Z_{p} Z_{t}$ systems.

Another interesting property of fission is the presence of asymmetric fission in low energy fission of actinides and pre-actinides due to the shell effects. There is strong evidence of multi-mode nature of fission, where a transition from asymmetric to symmetric mass division occurs as excitation energy is increased[16-18]. Mass distribution from such system will reflect contributions from both symmetric and asymmetric mass components. Hence presence of multi-mode fission makes the mass distribution different than single Gaussian at low energies.

In a series of experiments carried out at IUAC heavy ion accelerator facility [19], we have further extended the study of fission properties by measuring fragment mass and angular distributions from fission induced by medium light projectiles on spherical and deformed targets[20, 21]. The reactions were chosen to identify the role of deformation as well as mass asymmetry influencing the fission properties in these reactions.

\section{Experiments}

Experiments were performed using ${ }^{11} \mathrm{~B},{ }^{12} \mathrm{C}$ and ${ }^{19} \mathrm{~F}$ projectiles bombarding on ${ }^{206,208} \mathrm{~Pb}$ and ${ }^{238} \mathrm{U}$ targets at energies around the Coulomb barrier. We have measured the mass distribution of $\mathrm{FF}$ in reactions ${ }^{11} \mathrm{~B},{ }^{12} \mathrm{C}+{ }^{238} \mathrm{U},{ }^{19} \mathrm{~F}+$ ${ }^{206,208} \mathrm{~Pb}$, and both mass and angular distribution in ${ }^{19} \mathrm{~F}+$ ${ }^{238} \mathrm{U}$. Pulsed beam of $\sim 1.5 \mathrm{~ns}$ time width and $4 \mathrm{MHz}$ repetition rate were used in all time of flight measurements. Targets with typical thickness of $\sim 110 \mu \mathrm{g} / \mathrm{cm}^{2}$ were prepared on carbon backing $\sim 20 \mu \mathrm{g} / \mathrm{cm}^{2}$. For mass distribution measurement, a pair of multi-wire proportional counters (MWPCs) were used inside the $1.5 \mathrm{~m}$ diameter scattering chamber to collect FF in coincidence. Experimental setup and data analysis details are given in Ref.[20]. The data were analyzed to extract mass-angle correlation, mass ratio distribution and variance of mass distribution. From position and time signal, velocity components were reconstructed and mass ratio distribution was extracted from velocity components. Full momentum transfer condition was applied to separate transfer induced fission events. The extracted mass ratio $\left(M_{R}\right)$ distributions were fitted by Gaussian distribution at all energies and corresponding mass variance obtained.

\subsection{Mass distribution from reaction ${ }^{19} \mathbf{F}+{ }^{206,208} \mathbf{P b}$}

The reactions ${ }^{19} \mathrm{~F}+{ }^{206,208} \mathrm{~Pb}$ forms the light actinide compound nuclei ${ }^{225,227} \mathrm{~Pa}$. Mass distribution measurements were performed over the excitation energies $\mathrm{E}_{C N}$ ranging from 30 to $50 \mathrm{MeV}$. The results are reported in[20, 21]. Since both ${ }^{206,208} \mathrm{~Pb}$ targets are spherical, no deformation dependent effects are expected in these reactions. Our measurements showed mass distribution can be described by single symmetric Gaussian fit at all measured energies. A sample of measured mass distribution is shown in Fig. 1 for reaction ${ }^{19} \mathrm{~F}+{ }^{206} \mathrm{~Pb}$ at two beam energies, one at below the barrier $\frac{E_{C m}}{V_{B}}=0.93$ and other at above barrier energy $\frac{E_{C m}}{V_{B}}=1.1$. Though mass distribution could be fitted by single Gaussian, the mass variance obtained from the fit increased at lower energies. The data from ${ }^{19} \mathrm{~F}+{ }^{208} \mathrm{~Pb}$ also showed the same property. The plot of mass variance as a function of excitation energy for ${ }^{19} \mathrm{~F}+{ }^{208} \mathrm{~Pb}$ is given in figure $2 \mathrm{a}$. As the excitation energy decreases, the mass variance falls in relation to energy until it increases again at low excitation energy. Similar increase of mass variance at below barrier energy has been reported in system involving deformed target which has been attributed to onset of QF due to orientation effect[7, 8]. However in the present reaction, QF is minimal as there is no evidence of other signatures of QF here. No mass-angle correlation was observed in this reaction. The angular distribution data for ${ }^{19} \mathrm{~F}+{ }^{208} \mathrm{~Pb}$ as displayed in Fig. 2b already showed normal anisotropy as expected in complete fusionfission reaction[22]. A systematic evaluation of data from mass distribution of nearby light actinide nuclei showed that, the presence of asymmetric mass division at low excitation energy could be the reason for broadening of mass width [20]. The nuclei in this mass region showed multimode nature of fission, where there is a transition from symmetric mass division to asymmetric division as the excitation energy is decreased[16, 23, 24]. The origin of multi-mode fission has been attributed to the shell structure of fragments[25-28].
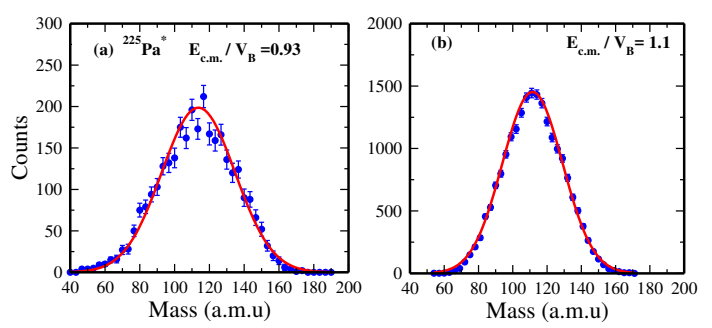

Figure 1. (Color online) The mass distribution of fission fragments from reaction ${ }^{19} \mathrm{~F}+{ }^{206} \mathrm{~Pb}$ (a) at below barrier energy $\frac{E_{C m}}{V_{B}}$ $=0.93$ and (b) above barrier energy $\frac{E_{C m}}{V_{B}}=1.1$. The solid line indicates best fit to data using single Gaussian function.

\subsection{Shell effects in mass distribution of ${ }^{12} \mathbf{C}+{ }^{238} \mathbf{U}$}

The importance of shell effects in fission mass distribution was highlighted in a recent paper where mass distribution from compound nuclei ${ }^{242-247} \mathrm{Cf}$ was compared against different entrance channels[29]. Mass distribution of reaction ${ }^{12} \mathrm{C}+{ }^{235} \mathrm{U}$ around barrier energies showed flat top distribution signifying contribution of more asymmetric fission components in this channel as compared to other reaction such as $\mathrm{S}+\mathrm{Pb}$. The mass-asymmetric component here was attributed to low excitation energy fission due 


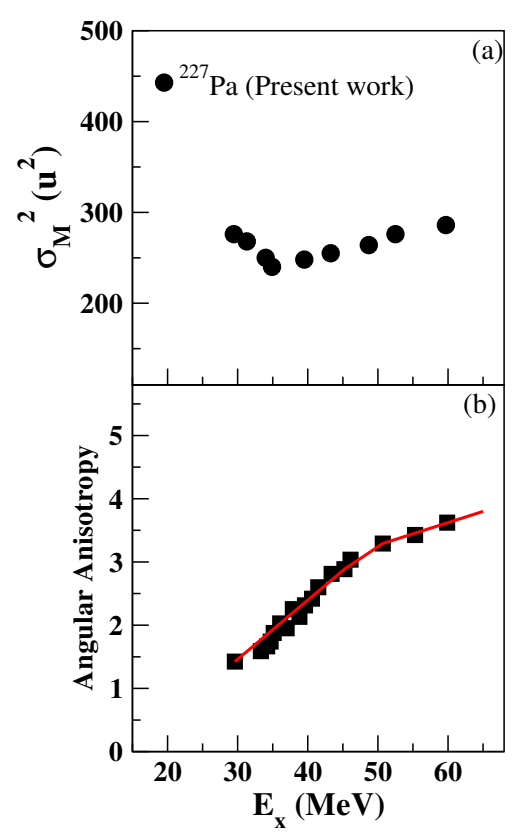

Figure 2. (Color online) (a) Mass variance $\left(\sigma_{M}^{2}\right)$ at various excitation energies in reaction ${ }^{19} \mathrm{~F}+{ }^{208} \mathrm{~Pb}$, from Ref.[20] and (b) the fission fragment angular anisotropy data from Ref.[22].The solid line in (b) corresponds to SSPM predictions.

to multi-chance fission. This observation has been validated with detailed theoretical model calculation using GEF code which predict the fraction of asymmetric fission considering shell effects at low excitation energies quite well[30]. From one of our earlier measurement at IUAC, we have already reported the mass distribution of reaction ${ }^{12} \mathrm{C}+{ }^{238} \mathrm{U}$ forming $\mathrm{CN}{ }^{250} \mathrm{Cf}$ and compared with another reaction ${ }^{18} \mathrm{O}+{ }^{232} \mathrm{Th}$ at around barrier energies[31]. A detailed re-analysis of low energy data from ${ }^{12} \mathrm{C}+{ }^{238} \mathrm{U}$ showed flat-topped non single Gaussian mass distribution in the fission of $\mathrm{CN}^{250} \mathrm{Cf}$ clearly demonstrating the presence of asymmetric fission arising from shell effects of fragments. The experimental mass distribution from reaction ${ }^{12} \mathrm{C}+{ }^{238} \mathrm{U}$ at lowest energy along with GEF prediction is depicted in figure 3. The prediction of GEF calculation when multi-chance fission is included matches closely with experimental data that can be described well by double Gaussian fitting.

\subsection{Fission study on ${ }^{11} \mathbf{B},{ }^{19} \mathbf{F}+{ }^{238} \mathbf{U}$ reactions}

The reaction ${ }^{11} \mathrm{~B}+{ }^{238} \mathrm{U} \longrightarrow{ }^{249} \mathrm{Bk}^{*}$ was performed using pulsed ${ }^{11} \mathrm{~B}$ beam with energy varying from $52 \mathrm{MeV}$ to 66 $\mathrm{MeV}$. Fission fragments were detected in coincidence by placing MWPC pairs at folding angles. The forward detector was placed $35 \mathrm{~cm}$ away from target and kept $40^{\circ}$ with respect to beam whereas the backside detector was placed $27 \mathrm{~cm}$ at $130^{\circ}$. For reaction ${ }^{19} \mathrm{~F}+{ }^{238} \mathrm{U} \longrightarrow{ }^{257} \mathrm{Md}^{*}$, ${ }^{19} \mathrm{~F}$ beam energy was varied from $92 \mathrm{MeV}$ to $120 \mathrm{MeV}$ and MWPC detectors were placed at corresponding folding angles. Event by event data analysis were performed using velocity reconstruction method as described before.

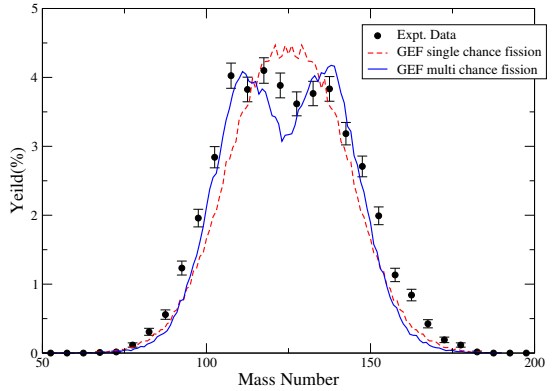

Figure 3. (Color online) Mass distribution of fission fragments from reaction ${ }^{12} \mathrm{C}+{ }^{238} \mathrm{U}$ at excitation energy $E_{C N}=34.2 \mathrm{MeV}$ along with GEF predicted distribution for single chance and multi chance fission.

\subsubsection{Mass distribution}

For both reactions, mass-angle-distribution(MAD) have been extracted at different energies and Fig. 4 shows a typical MAD plot for reaction ${ }^{19} \mathrm{~F}+{ }^{238} \mathrm{U}$ at below barrier energy corresponding to $\frac{E_{C m}}{V_{B}}=0.97$. The emission angle $\left(\theta_{\text {c.m. }}\right)$ span from $\approx 100^{\circ}$ to $160^{\circ}$. It is seen that that mass ratio is independent of $\theta_{c . m}$. suggesting there is no significant correlation between mass and angle of fragments in this reaction at any of the measured energies. No correlation is found in reaction ${ }^{11} \mathrm{~B}+{ }^{238} \mathrm{U}$ also.

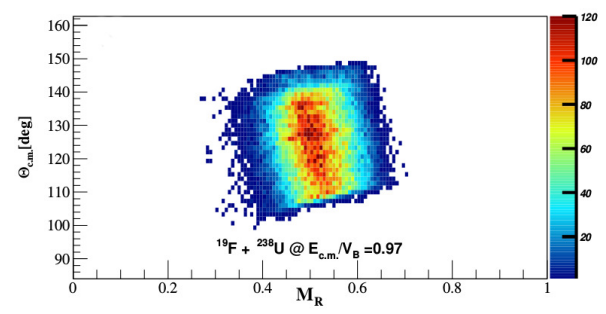

Figure 4. Mass-angle distribution of fragments from reaction ${ }^{19} \mathrm{~F}$ $+{ }^{238} \mathrm{U}$ at below barrier energy.

Fragment mass distribution measured in reactions ${ }^{11} \mathrm{~B}+{ }^{238} \mathrm{U}$ and ${ }^{19} \mathrm{~F}+{ }^{238} \mathrm{U}$ at two bombarding energies are shown in Fig. 5a-d. These energies correspond to below and above the barrier in respective cases. At all energies, the measured mass spectra from both reactions could be described by a single symmetric Gaussian centered around mass ratio 0.5. As the excitation energy in fissioning nuclei increases, the mass width increase as a function of temperature as expected. There is no significant deviation from symmetric Gaussian shape at any of the measured energy point which signify fully mass equilibrated fusionfission dominating in both reactions.

In earlier works, the nearby reaction ${ }^{16} \mathrm{O}+{ }^{238} \mathrm{U}$ showed skewness in mass distribution and enhancement in mass width at low excitation energies due to deformation dependent QF[7, 22]. Present measurement did not show any such behavior in reaction ${ }^{19} \mathrm{~F}+{ }^{238} \mathrm{U}$. The mass vari- 


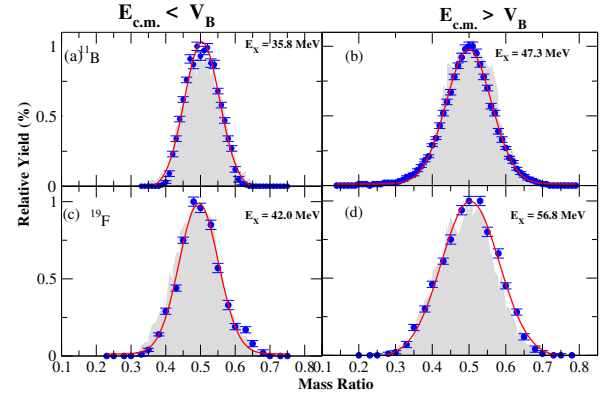

Figure 5. (a)-(b)Mass distribution from reaction ${ }^{11} \mathrm{~B}+{ }^{238} \mathrm{U}$ at below barrier energy(a) and above barrier energy (b). (c)-(d) mass distribution from ${ }^{19} \mathrm{~F}+{ }^{238} \mathrm{U}$ at below barrier (c) and above barrier energy (d). The solid line corresponds to best fit using single Gaussian and shaded region represent the results of GEMINI ${ }^{++}$ predictions.

Table 1. Entrance channel parameters for reactions used in this study. Columns shows the projectile, target, compound nucleus $(\mathrm{CN}), \alpha\left(=\frac{A_{T}-A_{P}}{A_{T}+A_{P}}\right), \alpha_{b g}$, fissility $(\chi), \mathrm{Z}_{P} \mathrm{Z}_{T}$.

\begin{tabular}{llllll}
\hline Projectile & $\mathrm{CN}$ & $\alpha$ & $\alpha_{b g}$ & $\chi$ & $\mathrm{Z}_{P} \mathrm{Z}_{T}$ \\
\hline${ }^{11} \mathrm{~B}$ & ${ }^{249} \mathrm{Bk}$ & 0.911 & 0.892 & 0.813 & 460 \\
${ }^{12} \mathrm{C}$ & ${ }^{250} \mathrm{Cf}$ & 0.904 & 0.896 & 0.823 & 552 \\
${ }^{16} \mathrm{O}$ & ${ }^{254} \mathrm{Fm}$ & 0.874 & 0.902 & 0.841 & 736 \\
${ }^{19} \mathrm{~F}$ & ${ }^{257} \mathrm{Md}$ & 0.852 & 0.905 & 0.849 & 828 \\
\hline
\end{tabular}

ance did not show anomalous enhancement at low excitation energy region. For these systems, the theoretical mass distribution has been simulated using statistical code GEMINI $^{++}$[32] which showed the predicted mass distribution closely matching with experimental data in both cases. We assume that the measured mass distribution in these reactions follow normal fusion-fission at all measured energies. The absence of any mass angle-correlation also suggest that the reactions are mainly dominated by fission events followed from mass equilibrated system. The entrance channel parameters for these reactions are shown in Table 1.

\subsubsection{Angular Distribution}

Angular distribution measurement of fragments in reaction ${ }^{19} \mathrm{~F}+{ }^{238} \mathrm{U}$ around barrier energy was performed using the hybrid detector telescope array (HyTAR) facility setup inside the scattering chamber. The array consist nine $\Delta E$ (gas ionization) and $\mathrm{E}$ (Silicon) detectors for particle identification based on differential energy loss method. The DC beam of ${ }^{19} \mathrm{~F}$ ions with energies ranging from $96 \mathrm{MeV}$ to $121 \mathrm{MeV}$ was used to bombard the ${ }^{238} \mathrm{U}$ target. The angular range covered from $70^{\circ}$ to $170^{\circ}$ with respect to beam direction. The data analysis was performed to determine the anisotropy in angular distribution.

\subsubsection{Angular anisotropy}

After applying the Jacobian for conversion of data from lab frame to centre-of mass frame, the angular anisotropy has been extracted from the ratio $\frac{W\left(180^{\circ}\right)}{W\left(90^{\circ}\right)}$ where $W\left(180^{\circ}\right)$ and $W\left(90^{\circ}\right)$ are the fission yields at $180^{\circ}$ and $90^{\circ}$ respectively. The experimental anisotropy has been then compared with anisotropy calculated according to the statistical saddle point model (SSPM). Figure 6 displays the ratio of measured anisotropy to theoretical anisotropy as a function of energy for present reaction ${ }^{19} \mathrm{~F}+{ }^{238} \mathrm{U}$ along with data from reaction ${ }^{16} \mathrm{O}+{ }^{238} \mathrm{U}[5]$. Similar to ${ }^{16} \mathrm{O}$ induced fission, anomalously large anisotropy is observed in present reaction also.

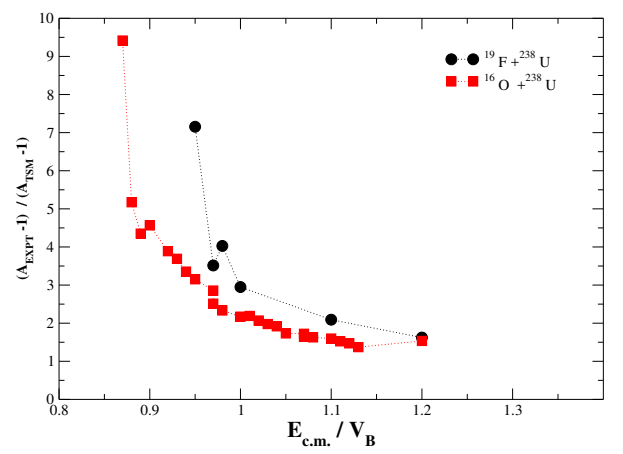

Figure 6. Ratio of measured anisotropy to theoretical anisotropy plotted as a function of energy. Solid circle represent data for reaction ${ }^{19} \mathrm{~F}+{ }^{238} \mathrm{U}$ and solid square corresponds data from ${ }^{16} \mathrm{O}+{ }^{238} \mathrm{U}[5]$

Anomalously large angular anisotropy has been observed in many other reactions using medium light projectiles (mass $A_{p}<24$ ) on various actinide targets ${ }^{232} \mathrm{Th},{ }^{238} \mathrm{U}$ and ${ }^{237} \mathrm{~Np}$ around barrier energies[9-11]. Though large angular anisotropy has been attributed to non-compound process such as PEF and QF, there is no clear evidence of which process influence the dynamics at various stages of fission process. Large angular anisotropy in ${ }^{19} \mathrm{~F}+{ }^{238} \mathrm{U}$ imply narrow $\mathrm{K}$ distribution suggesting incomplete shape equilibration, however there is no strong evidence of nonequilibration in mass degree of freedom. Symmetric mass distribution, normal mass width and absence of strong mass-angle correlation suggest that presence of QF may not be significant in reaction ${ }^{19} \mathrm{~F}+{ }^{238} \mathrm{U}$ at measured energies.

\subsection{Neutron multiplicity measurement}

Neutrons play a major role in the de-excitation process of heavy compound system. Neutron multiplicity is a sensitive observable for time scales and can be used as neutron clock. Since QF is fast process it is expected that average pre-scission neutron multiplicity will be lower in case of QF compared to fusion-fission[33]. Using mass gated neutron multiplicity measurements, extensive work has been carried out to discriminate fusion-fission and quasi- fission events based on the time scale involved in the process[34]. We have recently commissioned a large neutron detector array for studying the multiplicity of neutron emission in coincidence with fission fragments[35]. Using this facility experiment has been performed to extract mass gated neutron multiplicity from reaction ${ }^{19} \mathrm{~F}+{ }^{208} \mathrm{~Pb}$ at $110 \mathrm{MeV}$ 
Table 2. Pre-scission (Mpre) and post-scission(Mpost) neutron multiplicity gated by different mass split in reaction ${ }^{19} \mathrm{~F}+{ }^{208} \mathrm{~Pb}$ at $110 \mathrm{MeV}$ beam energy.

\begin{tabular}{lll}
\hline Mass Gate & Mpre & Mpost \\
\hline Symmetric & $2.29 \pm 0.20$ & $1.59 \pm 0.08$ \\
Asymmetric & $3.44 \pm 0.16$ & $1.05 \pm 0.06$ \\
\hline
\end{tabular}

beam energy. 50 neutron detectors and a pair of MWPCs were used to detect neutrons in coincidence with fission-fragments. Neutron-gamma discrimination was accomplished by pulse shape discrimination(PSD) technique. Figure 7 shows typical neutron-gamma discrimination from two dimensional plot between PSD and time of flight(TOF). The pre-scission and post-scission neutron multiplicities have been extracted using moving source fit applied to full set of experimental spectra. Global fitting for all neutron spectra were used to extract the parameters.

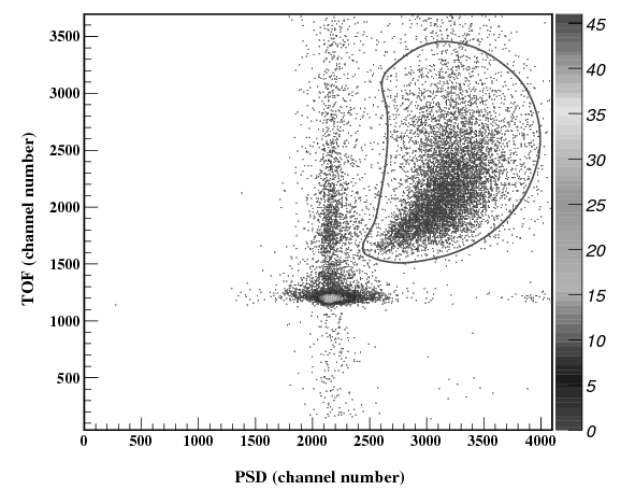

Figure 7. Neutron-gamma separation using PSD vs TOF. The gated region shows neutrons well separated.

Preliminary analysis shows that average pre-scission multiplicity is higher for asymmetric mass splits as compared to symmetric mass splits. Similar observation has been reported in reaction ${ }^{19} \mathrm{~F}+{ }^{209} \mathrm{Bi}$ at $108 \mathrm{MeV}$ beam energy[36]. The higher multiplicity for asymmetric mass region suggest the presence of multi-chance fission leading to asymmetric masses and more neutrons. Preliminary results are shown in Table 2 . The facility has been recently used for measuring mass gated neutron multiplicity in heavy systems using projectile ${ }^{48} \mathrm{Ti}$ on ${ }^{208} \mathrm{~Pb}$ target.

\section{Conclusion}

Fission fragment mass distributions have been measured for fission induced by projectiles such as ${ }^{11} \mathrm{~B},{ }^{12} \mathrm{C},{ }^{19} \mathrm{~F}$ on $\mathrm{Pb}$ and $\mathrm{U}$ targets in series of experiments performed at near barrier energies. Fission fragments were detected in coincidence using pair of MWPCs in time of flight setup and fragment's velocity components were extracted to generate mass distribution. For all reactions the mass distributions could be described by symmetric Gaussian. For ${ }^{225,227} \mathrm{~Pa}$ nuclei formed in fusion of ${ }^{19} \mathrm{~F}+{ }^{206,208} \mathrm{~Pb}$, the mass variance found to increase at below barrier energies.
Such enhancement in mass variance of at low energies have been observed in other light actinide nuclei ${ }^{224,226} \mathrm{Th}$ and ${ }^{228} \mathrm{U}$ where asymmetric and symmetric mass division were observed.

The role of deformation and mass asymmetry has been explored by measuring mass distribution in ${ }^{11} \mathrm{~B},{ }^{19} \mathrm{~F}+$ ${ }^{238} \mathrm{U}$ reaction and angular distribution in ${ }^{19} \mathrm{~F}+{ }^{238} \mathrm{U}$ reactions. In both systems mass distribution found to be symmetric and matches with $G E M I N I^{++}$model calculations. No strong evidence of mass asymmetry or deformation influencing the mass equilibration found in these reactions. However measured angular anisotropies of ${ }^{19} \mathrm{~F}$ $+{ }^{238} \mathrm{U}$ reaction showed anomalous large anisotropies deviating from SSPM predictions. This may suggest that in ${ }^{19} \mathrm{~F}+{ }^{238} \mathrm{U}$ there is evidence of fission events without equilibration in $\mathrm{K}$ degree of freedom but fully equilibrated in mass degree of freedom.

The authors wish to thank all collaborators, students, accelerator staff and Director, IUAC for their continuous support.

\section{References}

[1] J. Töke et al., Nucl.Phys. A 440,327(1985).

[2] B. Back et al., Phy.Rev.C, 32, 195(1985)

[3] D. Hinde et al., Phys.Rev.Lett., 101,092701(2008)

[4] A. C. Berriman et al., Nature 413, 144(2001)

[5] D.J. Hinde et al., Phy. Rev. C 53,1290(1996)

[6] C.J. Lin et al., Phy. Rev. C 85,014611(1996)

[7] K. Banerjee et al., Phys. Rev. C 83, 024605 (2011).

[8] T.K.Ghosh et al., Physics Letters B 627, 26 (2005).

[9] V.S. Ramamurthy and S.S. Kapoor, Phys. Rev. Lett. $54,178(1985)$

[10] Z. Liu et al, Phy. Lett. B 353,173(1995).

[11] J. P. Lestone et al., Phy. Rev. C 56, 2907(R) (1997)

[12] D. Hinde et al., Phys.Rev.Lett., 74, 1295(1995)

[13] B. Back et al., Phy.Rev. Lett, 46, 1068(1981)

[14] H.Q. Zhang, Phy. Rev. C 81,034611(2010).

[15] K. Nishio et al., Phys. Rev. Lett 93, 162701(2004)

[16] U. Brosa, S. Grossmann, A. Müller, Phys. Rep. 197, 167u (1990).

[17] Y. Nagame et al., Physics Letters B387, 26 (1996).

[18] K. H. Schmidt et al., Nucl. Phys. A 665, 221 (2000).

[19] G.K. Mehta and A.P. Patro, Nucl. Instr. and Meth. A 268, 334 (1988).

[20] R. Dubey et al., Physics Letters B 752, 338(2016).

[21] P. Sugathan, Journal of Physics: Conference Series, 755, 012007(2016).

[22] D. J. Hinde, et al., Nuclear Physics A685, 72 (2001).

[23] A. Turkevich and J. B. Niday, Phys. Rev. 84, 52 (1951).

[24] V.V. Pashkevich, Nucl. Phys. A 169, 275 (1971).

[25] P. Möller and J. R. Nix, Nucl. Phys. A229, 269 (1974).

[26] B.D. Wilkins, E.P. Steinberg, R.R. Chasman, Phys. Rev. C 14,1832 (1976).

[27] M.G. Mustafa, U. Mosel, H.W. Schmitt, Phys. Rev. C 7,1519 (1973). 
[28] V. Pokrovsky, M. G. Itkis, J. M. Itkis, et al., Phys.Rev. C62, 014615 (2000).

[29] J. Khuyagbaatar et al., Phys. Rev. C 91, 054608 (2015).

[30] B.Jurado and K.H. Schmidt, http://www.khserzhausen.de/GEF-2015-2.2.html,GEF code Version 2.2,(2015).
[31] C. Yadav et al., Phy.Rec. C 86, 034606(2012).

[32] R.J. Charity, Phy. Rev. C 82,014610(2010).

[33] L. Donadille et al., Nucl. Phys. A 656, 259 (1999)

[34] D. J. Hinde et al., Phys.Rev. C 30, 143 (1984).

[35] P. Sugathan et al, Pramana Journal of Physics 83, 807 (2014)

[36] L. M. Pant et al., Eur. Phys. J. A 16,43(2003) 